Article

\title{
Fixed Points for a Pair of $F$-Dominated Contractive Mappings in Rectangular $b$-Metric Spaces with Graph
}

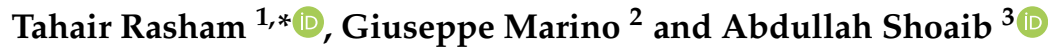 \\ 1 Department of Mathematics and Statistics, International Islamic University, H-10, Islamabad 44000, Pakistan \\ 2 Dipartimento di Matematica e Informatica, Universita della Calabria, 87036 Arcavacata di Rende (CS), Italy; \\ giuseppe.marino@unical.it \\ 3 Department of Mathematics and Statistics, Riphah International University, Islamabad 44000, Pakistan; \\ bdullahshoaib15@yahoo.com \\ * Correspondence: tahir_resham@yahoo.com
}

Received: 8 June 2019; Accepted: 15 July 2019; Published: 23 September 2019

\begin{abstract}
Recently, George et al. (in Georgea, R.; Radenovicb, S.; Reshmac, K.P.; Shuklad, S. Rectangular b-metric space and contraction principles. J. Nonlinear Sci. Appl. 2015, 8, 1005-1013) furnished the notion of rectangular b-metric pace (RBMS) by taking the place of the binary sum of triangular inequality in the definition of a b-metric space ternary sum and proved some results for Banach and Kannan contractions in such space. In this paper, we achieved fixed-point results for a pair of $F$-dominated mappings fulfilling a generalized rational $F$-dominated contractive condition in the better framework of complete rectangular $b$-metric spaces complete rectangular $b$-metric spaces. Some new fixed-point results with graphic contractions for a pair of graph-dominated mappings on rectangular $b$-metric space have been obtained. Some examples are given to illustrate our conclusions. New results in ordered spaces, partial $b$-metric space, dislocated metric space, dislocated $b$-metric space, partial metric space, $b$-metric space, rectangular metric spaces, and metric space can be obtained as corollaries of our results.
\end{abstract}

Keywords: fixed point; generalized $F$-contraction; $\alpha_{*}$-dominated mapping; graphic contractions

MSC: 46Txx; 47H10; 54H25

\section{Introduction and Preliminaries}

Fixed-point theory is a basic tool in functional analysis. Banach [1] has shown significant result for contraction mappings. Due to its significance, a large number of authors have proved newsworthy of this result (see [1-28]). In the sequel George et al. [2] furnished the notion of rectangular $b$-metric space (RBMS) by taking the place of the binary sum of triangular inequality in the definition of a $b$-metric space ternary sum and proved some results for Banach and Kannan contractions in such space. Further recent results on rectangular $b$-metric spaces can be seen in $[10,11]$. In this paper, we achieved fixed-point results for a pair of $\alpha$-dominated mappings fulfilling a generalized rational $F$-dominated contractive condition in complete rectangular $b$-metric spaces. Therefore, here, we investigate our results in a better framework of rectangular $b$-metric space. Some new fixed-point results with graphic contractions for a pair of graph-dominated mappings on rectangular $b$-metric space have been obtained. New results in ordered spaces, partial $b$-metric space, dislocated metric space, dislocated $b$-metric space, partial metric space, $b$-metric space, rectangular metric spaces, and metric space can be obtained as corollaries of our results. First, we give the precise definitions that we will use.

Definition 1 ([2]). Let $Z$ be a nonempty set and let $d_{l}: Z \times Z \rightarrow[0, \infty)$ be a function, called a rectangular $b$-metric (or simply $d_{l}$-metric), if there exists $b \geq 1$ such that the following conditions hold: 
(i) $d_{l}(g, p)=0$, if and only if $g=p$;

(ii) $d_{l}(g, p)=d_{l}(p, g)$;

(iii) $d_{l}(g, p) \leq b\left[d_{l}(g, q)+d_{l}(q, h)+d_{l}(h, p)\right]$ for all $g, p \in Z$ and all distinct points $q, h \in Z \backslash\{g, p\}$. The pair $\left(Z, d_{l}\right)$ is said a rectangular b-metric space (in short R.B.M.S) with coefficient $b$.

Definition 2 ([2]). Let $\left(Z, d_{l}\right)$ be a R.B.M.S.

(i) A sequence $\left\{g_{n}\right\}$ in $\left(Z, d_{l}\right)$ said to be Cauchy sequence if for each $\varepsilon>0$, there corresponds $n_{0} \in N$ such that for all $n, m \geq n_{0}$ we have $d_{l}\left(g_{m}, g_{n}\right)<\varepsilon$ or $\lim _{n, m \rightarrow \infty} d_{l}\left(g_{n}, g_{m}\right)=0$.

(ii) A sequence $\left\{g_{n}\right\}$ rectangular b-converges (for short $d_{l}$-converges) to $g$ if $\lim _{n \rightarrow \infty} d_{l}\left(g_{n}, g\right)=0$. In this case, $g$ is called a $d_{l}$-limit of $\left\{g_{n}\right\}$.

(iii) $\left(Z, d_{l}\right)$ is complete if every Cauchy sequence in $Z$ converges to a point $g \in Z$ for which $d_{l}(g, g)=0$.

Example 1 ([2]). Let $Z=N$ define $d: Z \times Z \rightarrow Z$ such that $d(u, v)=d(v, u)$ for all $u, v \in Z$ and

$$
d(u, v)=\left\{\begin{array}{c}
0, \text { if } u=v ; \\
10 \alpha, \text { if } u=1, v=2 ; \\
\alpha, \text { if } u \in\{1,2\} \text { and } v \in\{3\} ; \\
2 \alpha, \text { if } u \in\{1,2,3\} \text { and } v \in\{4\} ; \\
3 \alpha, \text { if } u \text { or } v \notin\{1,2,3,4\} \text { and } u \neq v ;
\end{array}\right.
$$

where $\alpha>0$ is a constant. Then $(Z, d)$ is a R.B.M.S with coefficient $b=2>1$, but $(Z, d)$ does not be a rectangular metric, since

$$
d(1,2)=10 \alpha>5 \alpha=d(1,3)+d(3,4)+d(4,2) .
$$

Definition 3 ([26]). Let $\left(Z, d_{l}\right)$ be a metric space, $S: Z \rightarrow P(Z)$ be a multivalued mapping and $\alpha: Z \times Z \rightarrow$ $[0,+\infty)$. Let $A \subseteq Z$, the mapping $S$ is said semi $\alpha_{*}$-admissible on $A$, if $\alpha(x, y) \geq 1$ implies $\alpha_{\star}(S x, S y) \geq 1$, for all $x \in A$, where $\alpha_{*}(S x, S y)=\inf \{\alpha(a, b): a \in S x, b \in S y\}$. When $A=Z$, we say that the $S$ is $\alpha_{*}$-admissible on $Z$. In the case in which $S$ is a single valued mapping, the previous definition becomes.

Definition 4. Let $\left(Z, d_{l}\right)$ be a R.B.M.S. Let $S: Z \rightarrow Z$ be a mapping and $\alpha: Z \times Z \rightarrow[0,+\infty)$. If $A \subseteq Z$, we say that the $S$ is $\alpha$-dominated on $A$, whenever $\alpha(i, S i) \geq 1$ for all $i \in A$. If $A=Z$, we say that $S$ is $\alpha$-dominated.

Definition 5 ([28]). Let $(Z, d)$ be a metric space. A mapping $H: Z \rightarrow Z$ is said to be an $A-$ contraction if there exists $\tau>0$ such that

$$
\forall j, k \in Z, d(H j, H k)>0 \Rightarrow \tau+A(d(H j, H k)) \leq A(d(j, k))
$$

with $A: \mathbb{R}_{+} \rightarrow \mathbb{R}$ real function which satisfies three assumptions:

(F1) $A$ is strictly increasing

(F2) For any sequence $\left\{\alpha_{n}\right\}_{n=1}^{\infty}$ of positive real numbers, $\lim _{n \rightarrow \infty} \alpha_{n}=0$ is equivalent to $\lim _{n \rightarrow \infty} A\left(\alpha_{n}\right)=$ $-\infty$;

(F3) There is $k \in(0,1)$ for which $\lim \alpha \rightarrow 0^{+} \alpha^{k} A(\alpha)=0$.

Example 2 ([19]). Let $Z=\mathbb{R}$. Define the mapping $\alpha: Z \times Z \rightarrow[0, \infty)$ by

$$
\alpha(x, y)=\left\{\begin{array}{c}
1 \text { if } x>y \\
\frac{1}{2} \text { otherwise }
\end{array}\right\} .
$$

Define the self-mappings $S, T: Z \rightarrow Z$ by $S x=\frac{x}{4}$, and Ty $=\frac{y}{2}$, where $x, y \in Z$. Suppose $x=3$ and $y=2$. As $3>2$, then $\alpha(3,2) \geq 1$. Now, $\alpha(S 3, T 2)=\frac{1}{2} \nsucceq 1$, this means the pair $(S, T)$ is not $\alpha$-admissible. Also, 
$\alpha(S 3, S 2) \ngtr 1$ and $\alpha(T 3, T 2) \ngtr 1$. This implies $S$ and $T$ are not $\alpha$-admissible individually. Now, $\alpha(x, S x) \geq 1$, for all $x \in Z$. Hence $S$ is $\alpha$-dominated mapping. Similarly it is clear that $\alpha(y, T y) \geq 1$ for all $x \in Z$. Hence it is clear that $S$ and $T$ are $\alpha$-dominated but not $\alpha$-admissible.

\section{Main Result}

Theorem 1. Let $\left(Z, d_{l}\right)$ be a complete R.B.M.S with coefficient $b \geq 1$. Let $\alpha: Z \times Z \rightarrow[0, \infty)$ be a function and $S, T: Z \rightarrow Z$ be the $\alpha$-dominated mappings on $Z$. Suppose that the following condition is satisfied:

There exist $\tau, \eta_{1}, \eta_{2}, \eta_{3}, \eta_{4}>0$ satisfying $b \eta_{1}+b \eta_{2}+(1+b) b \eta_{3}+\eta_{4}<1$ and a continuous and strictly increasing real function $F$ such that

$$
\tau+F\left(d_{l}(S e, T y)\right) \leq F\left(\begin{array}{c}
\eta_{1} d_{l}(e, y)+\eta_{2} d_{l}(e, S e) \\
+\eta_{3} d_{l}(e, T y)+\eta_{4} \frac{d_{l}^{2}(e, S e) \cdot d_{l}(y, T y)}{1+d_{l}^{2}(e, y)}
\end{array}\right)
$$

whenever $e, y \in\left\{g_{n}\right\}, \alpha(e, y) \geq 1$ and $d_{l}(S e, T y)>0$ "where the sequence $g_{n}$ is defined by $g_{0}$ arbitrary in $Z$, $g_{2 n+1}=S(T S)^{n} g_{0}$ and $g_{2 n}=(T S)^{n+1} g_{0}{ }^{\prime \prime}$. Then $\alpha\left(g_{n}, g_{n+1}\right) \geq 1$ for all $n \in \mathbb{N} \cup\{0\}$ and $\left\{g_{n}\right\} \rightarrow u \in Z$. Also, if the inequality (1) holds for $u$ and either $\alpha\left(g_{n}, u\right) \geq 1$ or $\alpha\left(u, g_{n}\right) \geq 1$ for all $n \in \mathbb{N} \cup\{0\}$, then $S$ and $T$ have a common fixed point $u$ in $\mathrm{Z}$.

Proof. Chose a point $g_{0}$ in $Z$ such that $g_{1}=S g_{0}$ and $g_{2}=T g_{1}$. Continuing this process we construct a sequence $\left\{g_{n}\right\}$ of points in $Z$ such that $g_{2 n+1}=S g_{2 n}$ and $g_{2 n+2}=T g_{2 n+1}$ for all for all $n \in \mathbb{N} \cup\{0\}$. Let $g_{1}, \cdots, g_{j} \in Z$ for some $j \in \mathbb{N}$. If $j$ is odd, then $j=2 \grave{i}+1$ for some $i \in \mathbb{N}$. Since $S, T: Z \rightarrow Z$ be the $\alpha$-dominated mappings on $Z$, so $\alpha\left(g_{2 i}, S g_{2 i}\right) \geq 1$ and $\alpha\left(g_{2 i+1}, T g_{2 i+1}\right) \geq 1$. As $\alpha\left(g_{2 i}, S g_{2 i}\right) \geq 1$, this implies $\alpha\left(g_{2 i}, S g_{2 i}\right)=\alpha\left(g_{2 i}, g_{2 i+1}\right) \geq 1$ where $g_{2 \grave{\imath}+1}=S g_{2 i}$. Now, by using inequality (1),

$$
\begin{aligned}
& \tau+F\left(d_{l}\left(g_{2 i+1}, g_{2 i+2}\right)\right) \leq \tau+F\left(d_{l}\left(S g_{2 i}, T g_{2 i+1}\right)\right) \\
& \leq F\left[\begin{array}{c}
\eta_{1} d_{l}\left(g_{2 i}, g_{2 i+1}\right)+\eta_{2} d_{l}\left(g_{2 i}, S g_{2 i}\right)+\eta_{3} d_{l}\left(g_{2 i}, T g_{2 i+1}\right) \\
+\eta_{4} \frac{d_{l}^{2}\left(g_{2 i}, S g_{2 i}\right) \cdot d_{l}\left(g_{2 i}, T g_{2 i+1}\right)}{1+d_{l}^{2}\left(g_{2 i}, g_{2 i+1}\right)}
\end{array}\right] \\
& \leq F\left[\begin{array}{c}
\eta_{1} d_{l}\left(g_{2 i}, g_{2 i+1}\right)+\eta_{2} d_{l}\left(g_{2 i}, g_{2 i ̀+1}\right)+b \eta_{3} d_{l}\left(g_{2 i}, g_{2 i ̀+1}\right) \\
+b \eta_{3} d_{l}\left(g_{2 i+1}, g_{2 i+2}\right)+\eta_{4} \frac{d_{l}^{2}\left(g_{2 i}, g_{2 i+1}\right) \cdot d_{l}\left(g_{2 i+1}, g_{2 i+2}\right)}{1+d_{l}^{2}\left(g_{2 i}, g_{2 i+1}\right)}
\end{array}\right] \\
& \leq F\left[\left(\eta_{1}+\eta_{2}+b \eta_{3}\right) d_{l}\left(g_{2 \grave{\imath}}, g_{2 \grave{i}+1}\right)+\left(b \eta_{3}+\eta_{4}\right) d_{l}\left(g_{2 \grave{i}+1}, g_{2 \grave{\imath}+2}\right)\right] \text {. }
\end{aligned}
$$

This implies

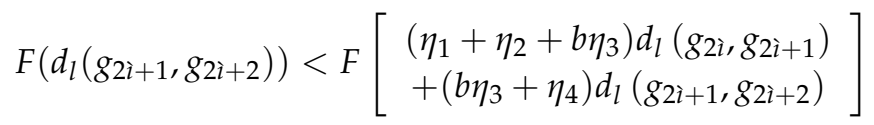

As $F$ is strictly increasing. Therefore, we have

$$
d_{l}\left(g_{2 \grave{\imath}+1}, g_{2 \grave{\imath}+2}\right)<\left[\begin{array}{c}
\left(\eta_{1}+\eta_{2}+b \eta_{3}\right) d_{l}\left(g_{2 \grave{i}}, g_{2 \grave{\imath}+1}\right) \\
+\left(b \eta_{3}+\eta_{4}\right) d_{l}\left(g_{2 \grave{i}+1}, g_{2 \grave{\imath}+2}\right) .
\end{array}\right]
$$

Which implies

$$
\begin{aligned}
\left(1-b \eta_{3}-\eta_{4}\right) d_{l}\left(g_{2 \grave{\imath}+1}, g_{2 \grave{\imath}+2}\right) & <\left(\eta_{1}+\eta_{2}+b \eta_{3}\right) d_{l}\left(g_{2 i}, g_{2 \grave{\imath}+1}\right) \\
d_{l}\left(g_{2 \grave{\imath}+1}, g_{2 \grave{\imath}+2}\right) & <\left(\frac{\eta_{1}+\eta_{2}+b \eta_{3}}{1-b \eta_{3}-\eta_{4}}\right) d_{l}\left(g_{2 \grave{\imath}}, g_{2 \grave{\imath}+1}\right) .
\end{aligned}
$$

Now, we note that by assumption of inequality (1) it immediately follows $\lambda=\frac{\eta_{1}+\eta_{2}+b \eta_{3}}{1-b \eta_{3}-\eta_{4}}<1$. Hence

$$
d_{l}\left(g_{2 \grave{\imath}+1}, g_{2 \grave{\imath}+2}\right)<\lambda d_{l}\left(g_{2 \grave{\imath}}, g_{2 \grave{\imath}+1}\right)<\lambda^{2} d_{l}\left(g_{2 \grave{\imath}-1}, g_{2 \grave{i}}\right)<\cdots<\lambda^{2 i+1} d_{l}\left(g_{0}, g_{1}\right) \text {. }
$$


Similarly, if $j$ is even, we have

$$
d_{l}\left(g_{2 i+2}, g_{2 i+3}\right)<\lambda^{2 i+2} d_{l}\left(g_{0}, g_{1}\right) .
$$

Now, we have

$$
d_{l}\left(g_{j}, g_{j+1}\right)<\lambda^{j} d_{l}\left(g_{0}, g_{1}\right) \text { for } j \in \mathbb{N} .
$$

Also $\alpha\left(g_{n}, g_{n+1}\right) \geq 1$ for all $n \in \mathbb{N} \cup\{0\}$. Now,

$$
d_{l}\left(g_{n}, g_{n+1}\right)<\lambda^{n} d_{l}\left(g_{0}, g_{1}\right) \text { for all } n \in \mathbb{N} .
$$

Now, for any positive integers $m, n(m>n)$, we have

$$
\begin{aligned}
& d_{l}\left(g_{n}, g_{m}\right) \leq b\left[d_{l}\left(g_{n}, g_{n+1}\right)+d_{l}\left(g_{n+1}, g_{n+2}\right)+d_{l}\left(g_{n+2}, g_{m}\right)\right] \\
& \leq b\left[d_{l}\left(g_{n}, g_{n+1}\right)+d_{l}\left(g_{n+1}, g_{n+2}\right)\right]+b^{2}\left[d_{l}\left(g_{n+2}, g_{n+3}\right)\right. \\
& \left.+d_{l}\left(g_{n+3}, g_{n+4}\right)+d_{l}\left(g_{n+4}, g_{m}\right)\right] \\
& \leq \quad b\left[\lambda^{n}+\lambda^{n+1}\right] d_{l}\left(g_{0}, g_{1}\right)+b^{2}\left[\lambda^{n+2}+\lambda^{n+3}\right] d_{l}\left(g_{0}, g_{1}\right) \\
& +b^{3}\left[\left[\lambda^{n+4}+\lambda^{n+5}\right] d_{l}\left(g_{0}, g_{1}\right)+\cdots\right. \\
& +b^{2 m-1} \lambda^{m-n} d_{l}\left(g_{0}, g_{1}\right), \\
& \leq b \lambda^{n}\left[1+b \lambda^{2}+b^{2} \lambda^{4}+\cdots\right] d_{l}\left(g_{0}, g_{1}\right) \\
& +b \lambda^{n+1}\left[1+b \lambda^{2}+b^{2} \lambda^{4}+\cdots\right] d_{l}\left(g_{0}, g_{1}\right) \\
& \leq \frac{1+\lambda}{1-b \lambda^{2}} b \lambda^{n} d_{l}\left(g_{0}, g_{1}\right) \text {. }
\end{aligned}
$$

As $\eta_{1}, \eta_{2}, \eta_{3}, \eta_{4}>0, b \geq 1$ and $b \eta_{1}+b \eta_{2}+(1+b) b \eta_{3}+\eta_{4}<1$, so $\left|b \lambda^{2}\right|<1$. Then, we have

$$
d_{l}\left(g_{n}, g_{m}\right)<\frac{1+\lambda}{1-b \lambda^{2}} b \lambda^{n} d_{l}\left(g_{0}, g_{1}\right) \rightarrow 0 \text { as } n \rightarrow \infty .
$$

Hence $\left\{g_{n}\right\}$ is a Cauchy sequence in $Z$. Since $\left(Z, d_{l}\right)$ is a complete metric space, so there exist $u \in Z$ such that $\left\{g_{n}\right\} \rightarrow u$ as $n \rightarrow \infty$, then

$$
\lim _{n \rightarrow \infty} d_{l}\left(g_{n}, u\right)=0
$$

By assumption, $\alpha\left(u, g_{n}\right) \geq 1$. Suppose that $d_{l}(u, S u)>0$, then there exists positive integer $k$ such that $d_{l}\left(T g_{2 n+1}, S u\right)>0$ for all $n \geq k$. For $n \geq k$, we have

$$
\begin{aligned}
& d_{l}(u, S u) \leq b\left[d_{l}\left(u, g_{n}\right)+d_{l}\left(g_{n}, g_{2 n+2}\right)+d_{l}\left(g_{2 n+2}, S u\right)\right] \\
& \leq b\left[d_{l}\left(u, g_{n}\right)+d_{l}\left(g_{n}, g_{2 n+1}\right)+d_{l}\left(T g_{2 n+1}, S u\right)\right] \\
& \leq b\left[d_{l}\left(u, g_{n}\right)+d_{l}\left(g_{n}, g_{2 n+1}\right)+d_{l}\left(S u, T g_{2 n+1}\right)\right] \\
&<b\left[\begin{array}{c}
d_{l}\left(u, g_{n}\right)+d_{l}\left(g_{n}, g_{2 n+1}\right)+\eta_{1} d_{l}\left(u, g_{2 n+1}\right) \\
+\eta_{2} d_{l}(u, S u)+\eta_{3} d_{l}\left(g_{2 n+1}, T g_{2 n+1}\right) \\
+\eta_{4} \frac{d_{l}(u, S u) \cdot d_{l}^{2}\left(g_{2 n+1}, T g_{2 n+1}\right)}{1+d_{l}^{2}\left(g_{2 n+1}, u\right)} .
\end{array}\right]
\end{aligned}
$$

Letting $n \rightarrow \infty$, and by using the inequalities (4) and (5) we get

$$
d_{l}(u, S u)<\eta_{3} d_{l}(u, S u)<d_{l}(u, S u),
$$


which is a contradiction. So, our supposition is wrong. Hence $d_{l}(u, S u)=0$. Similarly, by using the above inequlity

$$
\begin{aligned}
& d_{l}(u, T u) \leq b\left[d_{l}\left(u, g_{n}\right)+d_{l}\left(g_{n}, g_{2 n+1}\right)+d_{l}\left(g_{2 n+1}, T u\right)\right] \\
& d_{l}(u, T u) \leq b\left[d_{l}\left(u, g_{n}\right)+d_{l}\left(g_{n}, g_{2 n+1}\right)+d_{l}\left(g_{2 n}, T u\right)\right]
\end{aligned}
$$

we can get $d_{l}(u, T u)=0$. This shows that $u$ is a common fixed point of $S$ and $T$.

Example 3. Let $Z=A \cup B$, where $A=\left\{\frac{1}{n}: n \in\{2,3,4,5\}\right\}$ and $B=[1,2]$. Define $d_{l}: Z \times Z \rightarrow[0, \infty)$ such that defined by $d_{l}(x, y)=d_{l}(y, x)$ for $x, y \in Z$ and

$$
\left\{\begin{array}{c}
d_{l}\left(\frac{1}{2}, \frac{1}{3}\right)=d_{l}\left(\frac{1}{4}, \frac{1}{5}\right)=0.03 \\
d_{l}\left(\frac{1}{2}, \frac{1}{5}\right)=d_{l}\left(\frac{1}{3}, \frac{1}{4}\right)=0.02 \\
d_{l}\left(\frac{1}{2}, \frac{1}{4}\right)=d_{l}\left(\frac{1}{5}, \frac{1}{3}\right)=0.6 \\
d_{l}(x, y)=|x-y|^{2} \quad \text { otherwise. }
\end{array}\right.
$$

be the complete R.B.M.S with coefficient $b=4>1$ but $\left(Z, d_{l}\right)$ is neither a metric space nor a rectangular metric space. Take $\eta_{1}=\frac{1}{10}, \eta_{2}=\frac{1}{20}, \eta_{3}=\frac{1}{60}, \eta_{4}=\frac{1}{30}, \tau \in\left(0, \frac{12}{95}\right]$ then $b \eta_{1}+b \eta_{2}+(1+b) b \eta_{3}+\eta_{4}<1$, $\lambda=\frac{11}{56}$ and $F(x)=\ln x$. Consider the mapping $\alpha: Z \times Z \rightarrow[0, \infty)$ by

$$
\alpha(x, y)=\left\{\begin{array}{cc}
1 & \text { if } x>y \\
\frac{1}{2} \text { otherwise }
\end{array}\right\}
$$

Let $S, T: Z \rightarrow Z$ be defined as

$$
S x=\left\{\begin{array}{l}
\frac{1}{2} \text { if } x \in A \\
\frac{x}{4} \text { if } x \in B .
\end{array} \quad T x=\left\{\begin{array}{l}
\frac{1}{3} \text { if } x \in A \\
\frac{x}{4} \text { if } x \in B .
\end{array}\right.\right.
$$

As $\frac{1}{2}, \frac{1}{3} \in Z, \alpha\left(\frac{1}{2}, \frac{1}{3}\right)>1$ taking $F(x)=\ln x$, for any $\tau \in\left(0, \frac{12}{95}\right]$. Then $S$ and $T$ satisfy the condition of Theorem 1.

If, we take $S=T$ in Theorem 1 , then we are left with result.

Corollary 1. Let $\left(Z, d_{l}\right)$ be a complete R.B.M.S with coefficient $b \geq 1$. Let $\alpha: Z \times Z \rightarrow[0, \infty)$ be a function and $S: Z \rightarrow Z$ be the $\alpha$-dominated mapping on $Z$. Suppose that the following condition is satisfied:

There exist $\tau, \eta_{1}, \eta_{2}, \eta_{3}, \eta_{4}>0$ satisfying $b \eta_{1}+b \eta_{2}+(1+b) b \eta_{3}+\eta_{4}<1$ and a continuous and strictly increasing real function $F$ such that

$$
\tau+F\left(d_{l}(S e, S y)\right) \leq F\left(\begin{array}{c}
\eta_{1} d_{l}(e, y)+\eta_{2} d_{l}(e, S e) \\
+\eta_{3} d_{l}(e, S y)+\eta_{4} \frac{d_{l}^{2}(e, S e) \cdot d_{l}(y, S y)}{1+d_{l}^{2}(e, y)}
\end{array}\right),
$$

whenever $e, y \in\left\{g_{n}\right\}, \alpha(e, y) \geq 1$ and $d_{l}(S e, S y)>0$ "where the sequence $g_{n}$ is defined by $g_{0}$ arbitrary in $Z$, $g_{2 n+1}=S^{2 n} g_{0}$ ". Then $\alpha\left(g_{n}, g_{n+1}\right) \geq 1$ for all $n \in \mathbb{N} \cup\{0\}$ and $\left\{g_{n}\right\} \rightarrow u \in Z$. Also, if the inequality (6) holds for $u$ and either $\alpha\left(g_{n}, u\right) \geq 1$ or $\alpha\left(u, g_{n}\right) \geq 1$ for all $n \in \mathbb{N} \cup\{0\}$, then $S$ and $T$ have a common fixed point $u$ in $\mathrm{Z}$.

If, we take $\eta_{2}=0$ in Theorem 1, then we are left with the result.

Corollary 2. Let $\left(Z, d_{l}\right)$ be a complete R.B.M.S with constant $b \geq 1$. Let $\alpha: Z \times Z \rightarrow[0, \infty)$ be a function and $S, T: Z \rightarrow Z$ be the $\alpha$-dominated mappings on $Z$. Suppose that the following condition is satisfied: 
There exist $\tau, \eta_{1}, \eta_{3}, \eta_{4}>0$ satisfying $b \eta_{1}+(1+b) b \eta_{3}+\eta_{4}<1$ and a continuous and strictly increasing real function $F$ such that

$$
\tau+F\left(d_{l}(S e, T y)\right) \leq F\left(\begin{array}{c}
\eta_{1} d_{l}(e, y)+\eta_{3} d_{l}(e, T y) \\
+\eta_{4} \frac{d_{l}^{2}(e, S e) \cdot d_{l}(y, T y)}{1+d_{l}^{2}(e, y)}
\end{array}\right)
$$

whenever $e, y \in\left\{g_{n}\right\}, \alpha(e, y) \geq 1$ and $d_{l}(S e, T y)>0$ "where the sequence $g_{n}$ is defined by $g_{0}$ arbitrary in $Z$, $g_{2 n+1}=S(T S)^{n} g_{0}$ and $g_{2 n}=(T S)^{n+1} g_{0}{ }^{\prime \prime}$. Then $\alpha\left(g_{n}, g_{n+1}\right) \geq 1$ for all $n \in \mathbb{N} \cup\{0\}$ and $\left\{g_{n}\right\} \rightarrow u \in Z$. Also, if the inequality (7) holds for $u$ and either $\alpha\left(g_{n}, u\right) \geq 1$ or $\alpha\left(u, g_{n}\right) \geq 1$ for all $n \in \mathbb{N} \cup\{0\}$, then $S$ and $T$ have common fixed point $u$ in $Z$.

If, we take $\eta_{3}=0$ in Theorem 1 , then we are left with the result.

Corollary 3. Let $\left(Z, d_{l}\right)$ be a complete R.B.M.S with constant $b \geq 1$. Let $\alpha: Z \times Z \rightarrow[0, \infty)$ be a function and $S, T: Z \rightarrow Z$ be the $\alpha$-dominated mappings on $Z$. Suppose that the following condition is satisfied: There exist $\tau, \eta_{1}, \eta_{2}, \eta_{4}>0$ satisfying $b \eta_{1}+b \eta_{2}+\eta_{4}<1$ and a continuous and strictly increasing real function F such that

$$
\tau+F\left(d_{l}(S e, T y)\right) \leq F\left(\begin{array}{c}
\eta_{1} d_{l}(e, y)+\eta_{2} d_{l}(e, S e) \\
+\eta_{4} \frac{d_{l}^{2}(e, S e) \cdot d_{l}(y, T y)}{1+d_{l}^{2}(e, y)}
\end{array}\right),
$$

whenever $e, y \in\left\{g_{n}\right\}, \alpha(e, y) \geq 1$ and $d_{l}(S e, T y)>0$ "where the sequence $g_{n}$ is defined by $g_{0}$ arbitrary in $Z$, $g_{2 n+1}=S(T S)^{n} g_{0}$ and $g_{2 n}=(T S)^{n+1} g_{0}{ }^{\prime \prime}$. Then $\alpha\left(g_{n}, g_{n+1}\right) \geq 1$ for all $n \in \mathbb{N} \cup\{0\}$ and $\left\{g_{n}\right\} \rightarrow u \in Z$. Also, if the inequality (8) holds for $u$ and either $\alpha\left(g_{n}, u\right) \geq 1$ or $\alpha\left(u, g_{n}\right) \geq 1$ for all $n \in \mathbb{N} \cup\{0\}$, then $S$ and $T$ have common fixed point $u$ in $\mathrm{Z}$.

If, we take $\eta_{4}=0$ in Theorem 1 , then we are left with the result.

Corollary 4. Let $\left(Z, d_{l}\right)$ be a complete R.B.M.S with coefficient $b \geq 1$. Let $\alpha: Z \times Z \rightarrow[0, \infty)$ be a function and $S, T: Z \rightarrow Z$ be the $\alpha$-dominated mappings on $Z$. Suppose that the following condition is satisfied:

There exist $\tau, \eta_{1}, \eta_{2}, \eta_{3}, \eta_{4}>0$ satisfying $b \eta_{1}+b \eta_{2}+(1+b) b \eta_{3}+\eta_{4}<1$ and a continuous and strictly increasing real function $F$ such that

$$
\tau+F\left(d_{l}(S e, T y)\right) \leq F\left(\begin{array}{c}
\eta_{1} d_{l}(e, y)+\eta_{2} d_{l}(e, S e) \\
+\eta_{3} d_{l}(e, T y)
\end{array}\right)
$$

whenever $e, y \in\left\{g_{n}\right\}, \alpha(e, y) \geq 1$ and $d_{l}(S e, T y)>0$ "where the sequence $g_{n}$ is defined by $g_{0}$ arbitrary in $Z$, $g_{2 n+1}=S(T S)^{n} g_{0}$ and $g_{2 n}=(T S)^{n+1} g_{0}{ }^{\prime \prime}$, Then $\alpha\left(g_{n}, g_{n+1}\right) \geq 1$ for all $n \in \mathbb{N} \cup\{0\}$ and $\left\{g_{n}\right\} \rightarrow u \in Z$. Also, if the inequality (9) holds for $u$ and either $\alpha\left(g_{n}, u\right) \geq 1$ or $\alpha\left(u, g_{n}\right) \geq 1$ for all $n \in \mathbb{N} \cup\{0\}$, then $S$ and $T$ have a common fixed point $u$ in $\mathrm{Z}$.

\section{Fixed Points for Graphic Contractions}

Lastly, we give a realization of Theorem 1 in graph theory. Jachymski, [14], shown the particular case for contraction mappings on metric space with a graph. Hussain et al. [12], introduced the concept of graphic contractions and obtained a point fixed result. Further results on graphic contraction can be seen in $[8,21,27]$. Shang [25], discussed briefly basic notions of graph limit theory and fix some necessary notations and presented many interesting applications.

Definition 6. Let $Z$ be a nonempty set and $Q=(V(Q), W(Q))$ be a graph such that $V(Q)=Z, A \subseteq Z$. A mapping $S: Z \rightarrow Z$ is said to be a graph dominated on $A$ if $(p, q) \in W(Q)$, for all $q \in S p$ and $q \in A$.

Theorem 2. Let $\left(Z, d_{l}\right)$ be a complete R.B.M.S endowed with a graph $Q$ with coefficient $b \geq 1$. Let $S, T$ : $Z \rightarrow Z$ be two self mappings. Suppose that the following satisfy: 
(i) $S$ and $T$ are graph dominated on $Z$.

(ii) There exist $\tau, \eta_{1}, \eta_{2}, \eta_{3}, \eta_{4}>0$ satisfying $b \eta_{1}+b \eta_{2}+(1+b) b \eta_{3}+\eta_{4}<1$ and a continuous and strictly increasing real function $F$ such that

$$
\tau+F\left(H_{d_{l}}(S p, T q)\right) \leq F\left(\begin{array}{c}
\eta_{1} d_{l}(p, q)+\eta_{2} d_{l}(p, S p) \\
+\eta_{3} d_{l}(p, T q)+\eta_{4} \frac{d_{l}^{2}(p, S p) \cdot d_{l}(q, T q)}{1+d_{l}^{2}(p, q)}
\end{array}\right)
$$

whenever $p, q \in\left\{g_{n}\right\},(p, q) \in W(Q)$ and $d_{l}(S p, T q)>0$ "where the sequence $g_{n}$ is defined by $g_{0}$ arbitrary in $Z, g_{2 n+1}=S(T S)^{n} g_{0}$ and $g_{2 n}=(T S)^{n+1} g_{0}{ }^{\prime \prime}$. Then $\left(g_{n}, g_{n+1}\right) \in W(Q)$ and $\left\{g_{n}\right\} \rightarrow m^{*}$. Also, if the inequality (10) holds for $m^{*}$ and $\left(g_{n}, m^{*}\right) \in W(Q)$ or $\left(m^{*}, g_{n}\right) \in W(Q)$ for all $n \in \mathbb{N} \cup\{0\}$, then $S$ and $T$ have common fixed point $m^{*}$ in $\mathrm{Z}$.

Proof. Define, $\alpha: Z \times Z \rightarrow[0, \infty)$ by

$$
\alpha(p, q)=\left\{\begin{array}{rr}
1, & \text { if } p \in Z,(p, q) \in W(Q) \\
0, & \text { otherwise. }
\end{array}\right.
$$

As $S$ and $T$ are graph dominated on $Z$, then for $p \in Z,(p, q) \in W(Q)$ for all $q \in S p$ and $(p, q) \in W(Q)$ for all $q \in T p$. Therefore, $\alpha(p, q)=1$ for all $q \in S p$ and $\alpha(p, q)=1$ for all $q \in T p$. Hence $\alpha_{*}(p, S p)=1, \alpha_{*}(p, T p)=1$ for all $p \in Z$. Therefore, $S, T: Z \rightarrow Z$ are the $\alpha$-dominated mappings on $Z$. Moreover, inequality (10) can be written as

$$
\tau+F\left(H_{d_{l}}(S p, T q)\right) \leq F\left(\begin{array}{c}
\eta_{1} d_{l}(p, q)+\eta_{2} d_{l}(p, S p) \\
+\eta_{3} d_{l}(p, T q)+\eta_{4} \frac{d_{l}^{2}(p, S p) \cdot d_{l}(q, T q)}{1+d_{l}^{2}(p, q)}
\end{array}\right)
$$

whenever $p, q \in\left\{g_{n}\right\}, \alpha(p, q) \geq 1$ and $d_{l}(S p, T q)>0$. Also, (ii) holds. Then, by Theorem 1, we have $\left\{g_{n}\right\} \rightarrow s^{*} \in Z$. Now, $g_{n}, s^{*} \in Z$ and either $\left(g_{n}, s^{*}\right) \in W(Q)$ or $\left(s^{*}, g_{n}\right) \in W(Q)$ implies that either $\alpha\left(g_{n}, s^{*}\right) \geq 1$ or $\alpha\left(s^{*}, g_{n}\right) \geq 1$. Therefore, all the conditions of Theorem 1 are satisfied. Hence, by Theorem $1, S$ and $T$ have a common fixed point $s^{*}$ in $Z$ and $d_{l}\left(s^{*}, s^{*}\right)=0$.

\section{Conclusions}

In the present work, we have achieved fixed-point results for new generalized F-contraction for a more general class of $\alpha$-dominated mappings rather than $\alpha_{*}$-admissible mappings and for a weaker class of strictly increasing mapping $F$ rather than class of mappings $F$ used by Wordowski [28]. We introduced the concept of a pair of graph-dominated mappings and given a fixed-point existence result of a fixed point for graphic contractions. Our results generalized and extended many recent fixed-point results of Rasham et al. [16,20], Wordowski's result [28], Ameer et al. [6] and many classical results in the current literature (see $[4,7,9,13,17,18,23,24])$.

Author Contributions: Each author equally contributed to this paper, read and approved the final manuscript.

Funding: This paper is funded by Ministero dell'Istruzione, Universita e Ricerca (MIUR) and Gruppo Nazionale di Analisi Matemarica e Probabilita e Applicazioni (GNAMPA).

Acknowledgments: The authors are very grateful to the reviewers that with their suggestions have significantly improved the presentation of the paper.

Conflicts of Interest: The authors declare that they have no competing interests. 


\section{References}

1. Banach, S. Sur les opérations dans les ensembles abstraits et leur application aux equations itegrales. Fund. Math. 1922, 3, 133-181. [CrossRef]

2. Georgea, R.; Radenovicb, S.; Reshmac, K.P.; Shuklad, S. Rectangular b-metric space and contraction principles. J. Nonlinear Sci. Appl. 2015, 8, 1005-1013. [CrossRef]

3. Abbas, M.; Ali, B.; Romaguera, S. Fixed and periodic points of generalized contractions in metric spaces. Fixed Point Theory Appl. 2013, 2013, 243. [CrossRef]

4. Acar, Ö.; Durmaz, G.; Minak, G. Generalized multivalued F-contractions on complete metric spaces. Bull. Iran. Math. Soc. 2014, 40, 1469-1478.

5. Ali, M.U.; Kamranb, T.; Postolache, M. Solution of Volterra integral inclusion in b-metric spaces via new fixed point theorem. Nonlinear Anal. Model. Control 2017, 22, 17-30. [CrossRef]

6. Ameer, E.; Arshad, M. Two new generalization for F-contraction on closed ball and fixed point theorem with application. J. Math. Exten. 2017, 11, 1-24.

7. Arshad, M.; Khan, S.U.; Ahmad, J. Fixed point results for F-contractions involving some new rational expressions. J. Fixed Point Theory Appl. 2016, 11, 79-97. [CrossRef]

8. Bojor, F. Fixed point theorems for Reich type contraction on metric spaces with a graph. Nonlinear Anal. 2012, 75, 3895-3901. [CrossRef]

9. Chen, C.; Wen, L.; Dong, J.; Gu, Y. Fixed point theorems for generalized F-contractions in b-metric-like spaces. J. Nonlinear Sci. Appl. 2016, 9, 2161-2174. [CrossRef]

10. Ding, H.S.; Imdad, M.; Radenović, S.; Vujaković, J. On some fixed point results in b-metric, rectangular and b-rectangular metric spaces. Arab. J. Math. Sci. 2016, 22, 151-164.

11. Dung, N.V. The metrization of rectangular b-metric spaces. Topol. Appl. 2019, 261, 22-28. [CrossRef]

12. Hussain, N.; Al-Mezel, S.; Salimi, P. Fixed points for $\psi$-graphic contractions with application to integral equations. Abstr. Appl. Anal. 2013, 2013, 575869. [CrossRef]

13. Hussain, A.; Arshad, M.; Nazim, M. Connection of Ciric type F-contraction involving fixed point on closed ball. Ghazi Univ. J. Sci. 2017, 30, 283-291.

14. Jachymski, J. The contraction principle for mappings on a metric space with a graph. Proc. Am. Math. Soc. 2008, 1, 1359-1373. [CrossRef]

15. Kamran, T.; Postolache, M.; Ali, M.U.; Kiran, Q. Feng and Liu type F-contraction in b-metric spaces with application to integral equations. J. Math. Anal. 2016, 7, 18-27.

16. Mahmood, Q.; Shoaib, A.; Rasham, T.; Arshad, M. Fixed Point Results for the Family of Multivalued F-Contractive Mappings on Closed Ball in Complete Dislocated b-Metric Spaces. Mathematics 2019, 7, 56. [CrossRef]

17. Piri, H.; Kumam, P. Some fixed point theorems concerning F-contraction in complete metric spaces. Fixed Point Theory Appl. 2014, 2014, 210. [CrossRef]

18. Piri, H.; Rahrovi, S.; Morasi, H.; Kumam, P. Fixed point theorem for F-Khan-contractions on complete metric spaces and application to the integral equations. J. Nonlinear Sci. Appl. 2017, 10, 4564-4573. [CrossRef]

19. Rasham, T.; Shoaib, A.; Alamri, B.A.S.; Arshad, M. Multivalued Fixed Point Results for New Generalized F-Dominated Contractive Mappings on Dislocated Metric Space with Application. J. Funct. Spaces 2018, 2018, 4808764. [CrossRef]

20. Rasham, T.; Shoaib, A.; Hussain, N.; Arshad, M.; Khan, S.U. Common fixed point results for new Ciric-type rational multivalued $F$-contraction with an application. J. Fixed Point Theory. Appl. 2018, 20, 45. [CrossRef]

21. Rasham, T.; Shoaib, A.; Alamri, B.A.S.; Asif, A.; Arshad, M. Fixed Point Results for $\alpha_{*}-\psi$-Dominated Multivalued Contractive Mappings Endowed with Graphic Structure. Mathematics 2019, 7, 307. [CrossRef]

22. Rasham, T.; Shoaib, A.; Hussain, N.; Alamri, B.A.S.; Arshad, M. Multivalued Fixed Point Results in Dislocated b-Metric Spaces with Application to the System of Nonlinear Integral Equations. Symmetry 2019, 11, 40. [CrossRef]

23. Secelean, N.A. Iterated function systems consisting of F-contractions. Fixed Point Theory Appl. 2013, 2013, 277. [CrossRef]

24. Sgroi, M.; Vetro, C. Multi-valued F-contractions and the solution of certain functional and integral equations. Filomat 2013, 27, 1259-1268. [CrossRef] 
25. Shang, Y. Limit of a nonpreferential attachment multitype network model. Int. J. Mod. Phys. B 2017, 31, 1750026. [CrossRef]

26. Shoaib, A.; Hussain, A.; Arshad, M.; Azam, A. Fixed point results for $\alpha_{*}-\psi$-Ciric type multivalued mappings on an intersection of a closed ball and a sequence with graph. J. Math. Anal. 2016, 7, 41-50.

27. Tiammee, J.; Suantai, S. Coincidence point theorems for graph-preserving multi-valued mappings. Fixed Point Theory Appl. 2014, 2014, 70. [CrossRef]

28. Wardowski, D. Fixed point theory of a new type of contractive mappings in complete metric spaces. Fixed Point Theory Appl. 2012, 2012, 94. [CrossRef]

(C) 2019 by the authors. Licensee MDPI, Basel, Switzerland. This article is an open access article distributed under the terms and conditions of the Creative Commons Attribution (CC BY) license (http://creativecommons.org/licenses/by/4.0/). 